\title{
A new convenient synthesis of some novel 2,6-disubstituted-pyridine derivatives
}

\author{
Korany A. Ali \\ Applied Organic Chemistry Department, National Research Center, Cairo, Egypt \\ E-mail: kornykhlil@yahoo.com
}

\begin{abstract}
The versatile, hitherto unreported pyridine-2,6-bis-(3-oxo-3-propanenitrile) 2 was prepared by the Claisen condensation of pyridine-2,6-dicarboxylic acid ester $\mathbf{1}$ with acetonitrile in the presence of sodium hydride. Several new pyrazoles, isoxazoles, and pyrazolopyridazine derivatives have been synthesized by the reaction of pyridine-2,6-bis-(3-oxo-3-propanenitrile) 2 with bidentate nucleophilic reagents and hydrazonoyl chloride derivatives.
\end{abstract}

Keywords: Pyridine-2,6-bis-(3-oxo-3-propanenitrile), pyrazole, isoxazole, and hydrazonoyl chloride, pyrazolopyridazine

\section{Introduction}

Substituted 3-oxopropanenitriles represent a category of versatile synthetic intermediates. They provide miscellaneous building blocks in the synthesis of several types of heterocyclic ring systems based on a wide range of condensation and cyclization reactions. ${ }^{1-9}$ On the other hand, a great deal of interest has been focused on the synthesis of the functionalized pyridine derivatives due to their biological activities. ${ }^{10,11}$ For example, some 2-pyridine radicals are incorporated into the structures of cardiotonic agents such as milrinone ${ }^{12}$ and HIV-1 specific transcriptase inhibitors. ${ }^{13}$ Furthermore, pyrazoles have also important applications in medicinal chemistry. For example, they have emerged as analgesic and anti-inflammatory drugs. ${ }^{14,15}$ In view of these observations and in continuation of our work on the synthesis of heterocyclic systems for biological evaluations, ${ }^{16-19}$ we considered it attractive to synthesize some pyrazoles and isoxazoles incorporated into pyridine moiety at position 2 and 6 . Hitherto unrecorded pyridine-2, 6-bis-(3-oxo-3-propanenitrile) $\mathbf{2}$ was prepared, and used as a building block for the synthesis of the title compounds. 


\section{Results and Discussion}

3-Oxoalkanonitriles can be generally prepared via: (i) acylation of active nitriles in the presence of suitable basic catalysts; ${ }^{20-22}$ (ii) reacting $\alpha$-haloketones with cyanide ion, ${ }^{23}$ and iii) hydrolysis of $\beta$-enaminonitriles ${ }^{7}$. We decided to develop our synthesis of compound 2 via the Claisen condensation of 2,6-pyridine dicarboxylic ethyl ester 1 with acetonitrile in dry THF in the presence of sodium hydride as strong base (Scheme 1).<smiles>CCOC(=O)c1cccc(C(=O)OCC)n1</smiles>

1

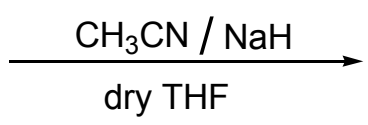

dry THF

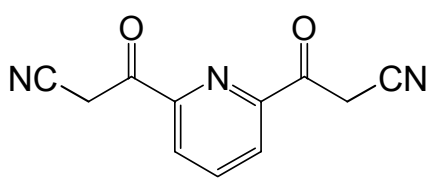

2

\section{Scheme 1}

The unique reactivity of oxonitriles arises from the closeness of two strongly electron withdrawing groups. In general, the reactions of saturated oxonitriles reflect the high acidity of protons adjacent to carbonyl and nitrile group. Moreover, the keto and cyano functions are suitably situated to facilitate reactions with common bidentate reagents. ${ }^{24}$

Treatment of pyridine-2,6-bis(3-oxo-3-propanenitrile) 2 with hydrazine hydrate in acetic acid, at room temperature, afforded a single product identified as 2,6-bis(5-amino- $1 \mathrm{H}$-pyrazol-3yl)pyridine 3. Compound 2 reacted with phenyl hydrazine in ethanol in the presence of a catalytic amount of piperidine, to yield a product which may be formulated as $\mathbf{5}$ or its isomer $\mathbf{6}$ (Scheme 2). The formation of compound $\mathbf{5}$ is assumed to proceed via condensation of the unsubstituted nitrogen atom of phenyl hydrazine with the carbonyl function in the oxonitriles 2 followed by intramolecular cyclization. Structure $\mathbf{5}$ was considered for a product based on similarity to the well established behavior of acyl and aroyl acetonitrile on the reaction with aryl hydrazines. ${ }^{25}$ Structure 5 was further confirmed by the treatment of oxonitriles $\mathbf{2}$ with phenyl hydrazine at room temperature to give the open structure $\mathbf{4}$ which undergoes intramolecular cyclization when refluxed in ethanol in the presence of pipredine to afford a compound identical in all respects (TLC, mp, and spectra) with those of compound 5 (Scheme 2). Treatment of compound 5 with dimethylformamide-dimethylacetal (DMF-DMA) under reflux, afforded 2,6 bis- $N, N$-dimethyl- $N$ '-(1-phenyl-1 $H$-pyrazol-5-yl)formamide-3-yl]pyridine 7 (Scheme 2). The ${ }^{1} \mathrm{H}$ NMR spectrum of the later compound revealed two singlets at $\delta 2.98$ and 3.11 due to methyl protons in addition to a singlet signals at $\delta 6.75$ and 7.90 due to methylidene and pyrazole protons, respectively. in addition to a multiplet at $\delta 7.32-7.65$ and $\delta 7.95-8.02$ due to aromatic and pyridine protons, respectively. Compound 2 reacted similarly with hydroxylamine to afford the corresponding 5-aminoisoxazole derivative 8 (Scheme 2). It should be noted here that, the reaction of 3-oxoalkanonitrile with hydroxylamine hydrochloride has been reported by Elnagdi et al. ${ }^{26}$ to yield amidoximes, that cyclized into 3 -aminoisoxazoles. 

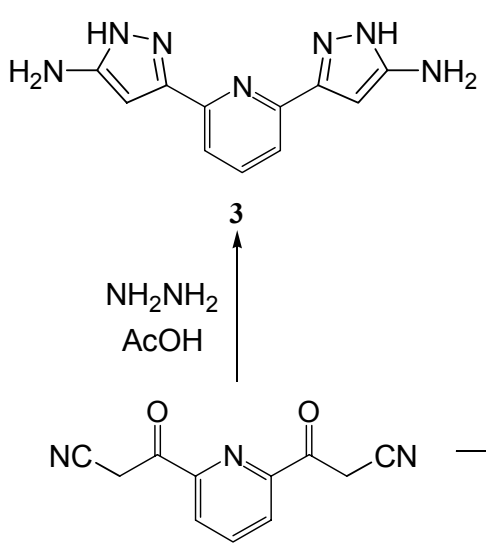

2

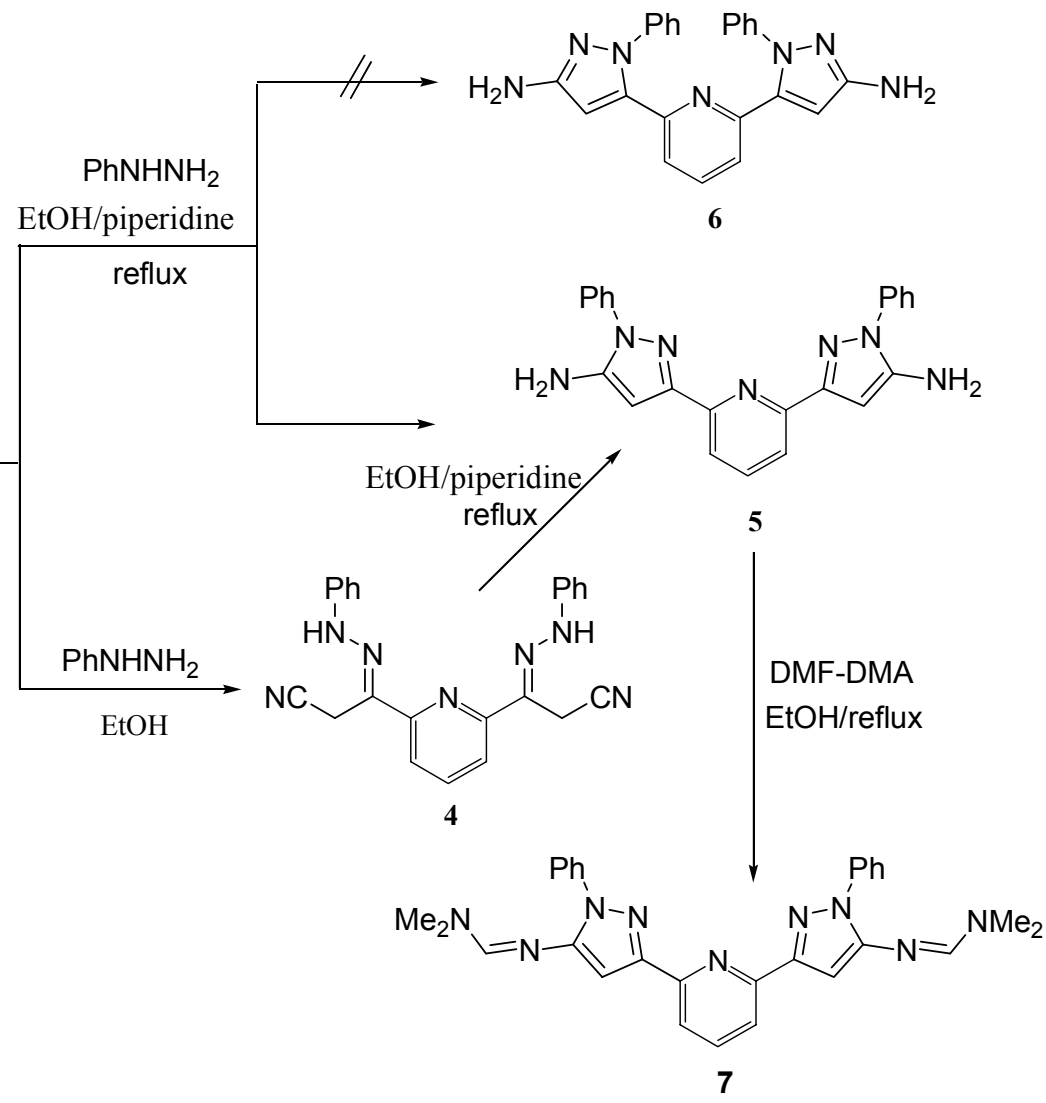

\section{Scheme 2}

The reactivity of compound $\mathbf{2}$ towards a variety of hydrazonoyl chlorides was also examined. Thus, treatment of compound $\mathbf{2}$ with ( $C$-acetyl-, $C$-ethoxycarbonyl, or $C$-acetanilido-)- $N$ arylhydrazonoyl chlorides 9a-g and sodium ethoxide, in absolute ethanol furnished, in each case, only one isolable product. The identities of the isolated products were assigned as the pyrazole derivatives 11a-g on the basis of their elemental analysis and spectral data (Scheme 3). For example, the IR spectra of compounds 11a-g showed, in each case, the presence of nitrile and carbonyl stretching bands near 2200 and $1700 \mathrm{~cm}^{-1}$ respectively, and revealed the lack of a band characteristic for amino group [see Exp. Part]. The $1 \mathrm{H}$ NMR spectrum of 11c taken as an example of the series synthesized, displayed signal at $\delta 2.40$ due to two acetyl groups, aromatic multiplet in the region $\delta 7.30-7.48$, in addition to a multiplet at $\delta 7.95-8.03$ due to pyridine protons and revealed the lack of a band characteristic for amino group. The possibilities of formation of the products 12a-g are excluded on the basis of the spectral data and the chemical behavior of the reaction products with hydrazine hydrate. The corresponding pyrazolopyridazine derivative 13a was obtained by the reaction of acetyl pyrazole 11a with an excess of hydrazine upon refluxing in ethanol for $5 \mathrm{~h}$. The lack of nitrile functions in the IR spectra of the isolated products supported the formation of structure 13a (Scheme 3). 


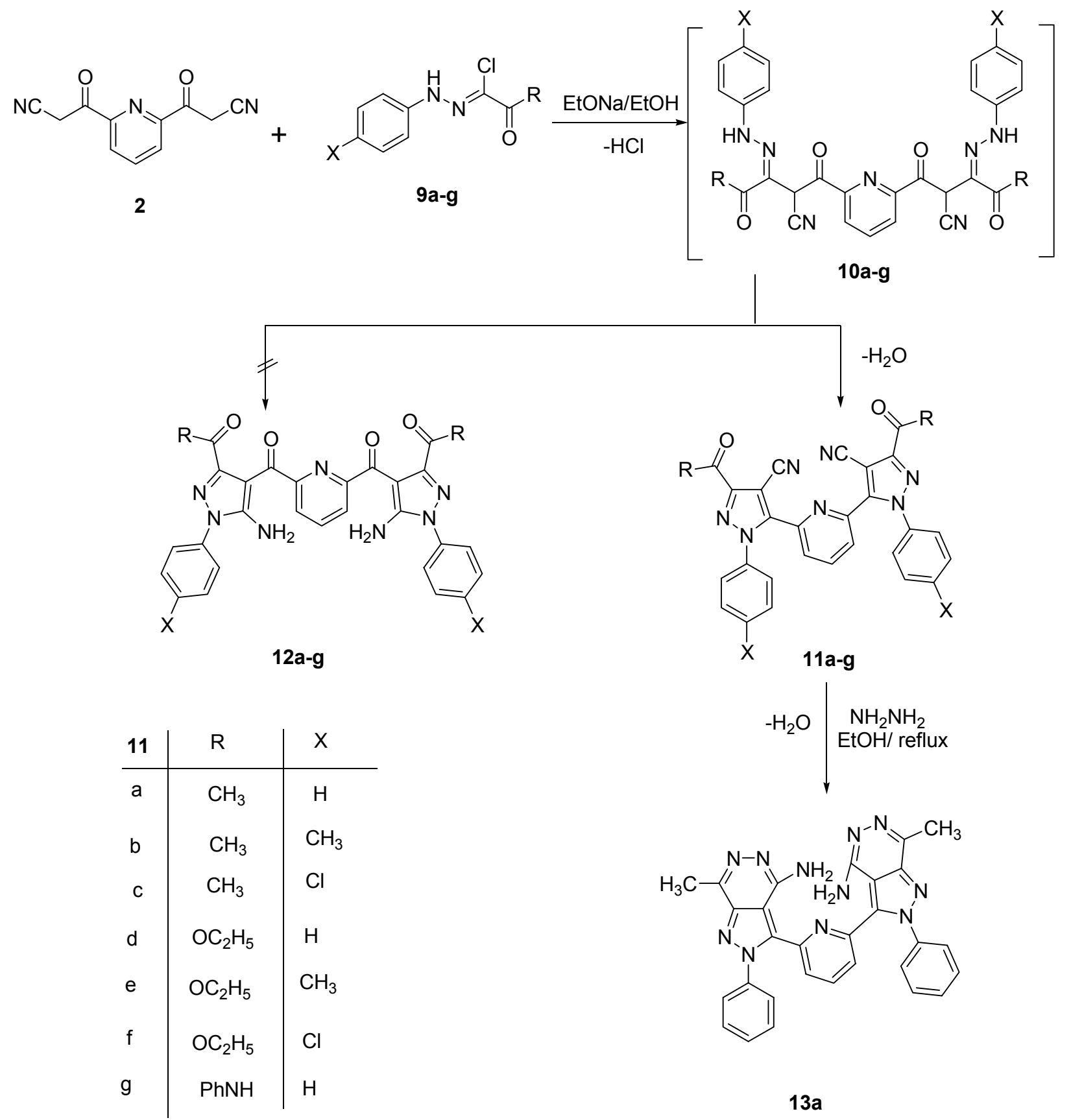

Scheme 3

\section{Experimental Section}

General. All melting points were measured on Electro thermal IA 9000 series digital melting point apparatus. The IR spectra were recorded in potassium bromide discs on a Pye Unicam SP 3300 and Shimadzu FT IR 8101 PC infrared spectrophotometer. The NMR Spectra were 
recorded at $270 \mathrm{MHz}$ on a Varian Mercury VX-300 NMR spectrometer. ${ }^{1} \mathrm{H}$ NMR (300 MHz) and ${ }^{13} \mathrm{C}$ NMR $(75.46 \mathrm{MHz})$ were run in deuterated chloroform $\left(\mathrm{CDCl}_{3}\right)$ or dimethylsulphoxide $\left(\mathrm{DMSO}-d_{6}\right)$. Chemical shifts were related to that of the solvent. Mass Spectra were recorded on a Shimadzu GCMS-QP1000 EX mass spectrometer at $70 \mathrm{eV}$. Elemental analyses were carried out at the Microanalytical Centre of Cairo University, Giza, Egypt. All reactions were followed by TLC (Silica gel, Aluminum Sheets $60 \mathrm{~F}_{254}$, Merck). Hydrazonoyl chlorides 9a-g ${ }^{27-29}$ were prepared as reported in the literature.

Pyridine-2,6-bis-(3-oxo-3-propanenitrile) (2). To a mixture of 2,6-pyridine dicarboxylic ethyl ester (1) (4.46 g, $20 \mathrm{mmol})$ and acetonitrile $(2.70 \mathrm{~mL}, 50 \mathrm{mmol})$, in dry THF $(50 \mathrm{~mL})$, was added sodium hydride ( $4 \mathrm{~g}, 50 \%)$. The reaction mixture was refluxed for $2 \mathrm{~h}$, and then allowed to cool. The solid that precipitated was filtered off, washed with diethyl ether and dried. The crude product was dissolved in water $(30 \mathrm{~mL})$ and the resulting alkaline solution was treated with concentrated hydrochloric acid until it becomes slightly acidic ( $\mathrm{pH}$ 5). The precipitated solid product was filtered off, washed with water, dried and finally recrystallized to afford pyridine2,6-bis-(3-oxo-3-propanenitrile) (2). Yield $80 \%$; brown crystals (from ethanol/dioxan); mp185$186{ }^{\circ} \mathrm{C}$. IR (KBr): $v 2264(\mathrm{CN}), 1720(\mathrm{C}=\mathrm{O}) \mathrm{cm}^{-1} .{ }^{1} \mathrm{H}$ NMR (DMSO- $\left.d_{6}\right): \delta 3.74\left(\mathrm{~s}, 2 \mathrm{H}, \mathrm{CH}_{2}\right)$, 8.57-8.24 (m, 3H, pyridine). ${ }^{13} \mathrm{C}$ NMR (DMSO- $d_{6}$ ): $\delta 29.6,116.4,123.7,126.4,165.7,190.7$; MS m/z (\%): $213\left[\mathrm{M}^{+}\right]$(75), 145 (100). Anal. Calcd for $\mathrm{C}_{11} \mathrm{H}_{7} \mathrm{~N}_{3} \mathrm{O}_{2}$ (213.19): C, $61.97 ; \mathrm{H}, 3.31 ; \mathrm{N}$, 19.71. Found: C, $61.94 ; \mathrm{H}, 3.34 ; \mathrm{N}, 19.75$.

2,6-Bis(5-amino-1 H-pyrazol-3-yl)pyridine (3). Hydrazine hydrate $(80 \%, 2 \mathrm{~mL})$ was added to a stirred solution of compound $2(0.21 \mathrm{~g}, 1 \mathrm{mmol})$ dissolved in acetic acid $(10 \mathrm{~mL})$. Stirring was continued for $7 \mathrm{~h}$ at room temperature and the obtained solid was filtered off, washed with cold water, dried and finally recrystallized to afford compound 3. Yield $75 \%$; white crystals (from ethanol/DMF); mp 261-262 ${ }^{\circ} \mathrm{C}$. IR (KBr): v 3302, 3275, $3200(\mathrm{NH}),\left(\mathrm{NH}_{2}\right) \mathrm{cm}^{-1} .{ }^{1} \mathrm{H}$ NMR (DMSO- $d_{6}$ ): $\delta 4.01$ (br s, $4 \mathrm{H}, \mathrm{D}_{2} \mathrm{O}$-exchangeable, $2 \mathrm{NH}_{2}$ ), $6.09(\mathrm{~s}, 2 \mathrm{H}$, pyrazole), 7.50-7.81 (m, $3 \mathrm{H}$, pyridine), 10.95 (s, 1H, $\mathrm{D}_{2} \mathrm{O}$-exchangeable, $\mathrm{NH}$ ). $\mathrm{MS} \mathrm{m} / z$ (\%): $241\left[\mathrm{M}^{+}\right]$(100), 212 (10), 185 (25), 76 (13). Anal. Calcd for $\mathrm{C}_{11} \mathrm{H}_{11} \mathrm{~N}_{7}$ (241.11): C, 54.76; H, 4.60; N, 40.64. Found: C, $54.81 ; \mathrm{H}, 4.65 ; \mathrm{N}, 40.61$.

E,E-2,6-Bis[3-(2-phenylhydrazino)-3-propanenitrile]pyridine (4). A mixture of compound 2 $(0.21 \mathrm{~g}, 1 \mathrm{mmol})$, phenylhydrazine $(0.30 \mathrm{~g}, 3 \mathrm{mmol})$, in ethanol $(10 \mathrm{~mL})$, was stirred at room temperature for $3 \mathrm{~h}$. The formed solid was filtered off, dried and recrystallized to afford compound 6. Yield 85\%; white powder (from ethanol); mp 217-218 ${ }^{\circ} \mathrm{C}$. IR (KBr): $v 3319(\mathrm{NH})$, $2240(\mathrm{CN}) \mathrm{cm}^{-1} .{ }^{1} \mathrm{H}$ NMR (DMSO- $\left.d_{6}\right): \delta 4.34\left(\mathrm{~s}, 4 \mathrm{H}, 2 \mathrm{CH}_{2}\right.$ ), 7.15-7.34 (m, 10H, ArH's), 7.678.03 (m, 3H, pyridine), 10.27 (s, 1H, $\mathrm{D}_{2} \mathrm{O}$-exchangeable, $\left.\mathrm{NH}\right)$. MS m/z (\%): $393\left[\mathrm{M}^{+}\right](100)$. Anal. Calcd for $\mathrm{C}_{23} \mathrm{H}_{19} \mathrm{~N}_{7}$ (393.44): C, 70.21; H, 4.87; N, 24.92. Found: C, 70.25; H, 4.84; N, 24.89.

\section{2,6-Bis[5-amino-1-phenyl-1 $H$-pyrazol-3-yl]pyridine(5)}

Method A. To a solution of $2(0.21 \mathrm{~g}, 1 \mathrm{mmol})$, in ethanol $(10 \mathrm{~mL})$, were added phenylhydrazine $(0.30 \mathrm{~g}, 3 \mathrm{mmol})$ and few drops of piperidine. The reaction mixture was refluxed for $5 \mathrm{~h}$ then 
allowed to cool. The formed solid was filtered off, dried and recrystallized to afford compound $\mathbf{5}$ (yield 90\%)

Method B. To a solution of $4(0.21 \mathrm{~g}, 0.05 \mathrm{mmol})$, in ethanol $(10 \mathrm{~mL})$, were added few drops of piperidine. The reaction mixture was refluxed for $3 \mathrm{~h}$ then allowed to cool. The formed solid was filtered off, dried and recrystallized afford a product identical in all respects $(\mathrm{mp}$, mixed mp, TLC, IR and mass spectra with that obtained via method A (yield 95\%). Yellow needles (from ethanol/dioxan); mp 241-242 ${ }^{\circ} \mathrm{C}$. IR (KBr): $v$ 3321, $3216\left(\mathrm{NH}_{2}\right) \mathrm{cm}^{-1} .{ }^{1} \mathrm{H}$ NMR (DMSO- $\left.d_{6}\right): \delta$ $5.48\left(\mathrm{~s}, 4 \mathrm{H}, \mathrm{D}_{2} \mathrm{O}\right.$-exchangeable $\left.2 \mathrm{NH}_{2}\right), 6.19$ (s, 2H, pyrazole), $7.53-7.67$ (m, 10H, ArH's), 7.858.01 (m, 3H, pyridine). ${ }^{13} \mathrm{C}$ NMR (DMSO- $\left.d_{6}\right): \delta 89.1,118.3,123.7,127.2,129.8,137.4,139.7$, 148.7, 151.6, 152.2; MS m/z (\%):393 [ $\left.\mathrm{M}^{+}\right]$(100), 77 (90.6). Anal. Calcd for $\mathrm{C}_{23} \mathrm{H}_{19} \mathrm{~N}_{7}(393.44)$ : C, 70.21; H, 4.87; N, 24.92. Found: C, 70.15; H, 4.87; N, 24.95.

2,6 Bis[ $N, N$-dimethyl- $N$ '-(1-phenyl-1 $H$-pyrazol-5-yl)formamide-3-yl]pyridine (7). A solution of compound 5 (0.39 g, $1 \mathrm{mmol})$ and dimethylformamide-dimethylacetal (DMF-DMA) (0.24 g, 2 mmol), in absolute ethanol $(10 \mathrm{~mL})$, was refluxed for $4 \mathrm{~h}$, and then allowed to cool. The solid product was filtered off, washed with ethanol, dried and crystallized to afford compound 7. Yield $85 \%$; Yellow needles (from ethanol/dioxan); mp 202-203 ${ }^{\circ} \mathrm{C}$. IR $(\mathrm{KBr}): v 1634(\mathrm{C}=\mathrm{N}) \mathrm{cm}^{-1} .{ }^{1} \mathrm{H}$ NMR (DMSO- $\left.d_{6}\right): \delta 2.98,3.11\left(2 \mathrm{~s}, 12 \mathrm{H}, 4 \mathrm{CH}_{3}\right), 6.75(\mathrm{~s}, 2 \mathrm{H}$, pyrazole-H-4), 7.30-7.48(m, 10H, ArH's), 7.90 (s, 2H, CH enamine), 7.95-8.03 (m, 3H, pyridine). ${ }^{13} \mathrm{C}$ NMR (DMSO- $d_{6}$ ): $\delta 34.5$, $40.1,90.1,118.5,123.6,125.8,128.3,128.4,136.7,140.4,151.7,152.4,152.8,154.8 ; \mathrm{MS} \mathrm{m} / z$ (\%):503 [M+] (100), 371 (15),186 (69),77(63). Anal. Calcd for $\mathrm{C}_{29} \mathrm{H}_{29} \mathrm{~N}_{9}$ (503.60): C, 69.16; $\mathrm{H}$, 5.80; N, 25.03. Found: C, 69.12; H, 5.84; N, 25.06

2,6-Bis(5-aminoisoxazol-3-yl)pyridine (8). To a solution of 2 (0.42 g, 2 mmol), in ethanol (10 $\mathrm{mL})$, were added hydroxylamine hydrochloride $(0.28 \mathrm{~g}, 4 \mathrm{mmol})$ and ammonium acetate $(0.6 \mathrm{~g})$. The reaction mixture was refluxed for $4 \mathrm{~h}$, and then poured onto ice cold water. The resulting solid was filtered off, washed with cold water, dried and recrystallized to afford 2,6-Bis[5aminoisoxazol-3-yl]pyridine 8. Yield $75 \%$; brown solid (from ethanol/dioxan); mp 232-234 ${ }^{\circ} \mathrm{C}$. IR (KBr): $v$ 3310, $3193\left(\mathrm{NH}_{2}\right) \mathrm{cm}^{-1} .{ }^{1} \mathrm{H}$ NMR (DMSO- $\left.d_{6}\right): \delta 5.52\left(\mathrm{~s}, 4 \mathrm{H}, \mathrm{D}_{2} \mathrm{O}\right.$-exchangeable, $\left.2 \mathrm{NH}_{2}\right), 6.85\left(\mathrm{~s}, 2 \mathrm{H}\right.$, isoxazole-H-4), 7.90-8.05 (m, 3H, pyridine). ${ }^{13} \mathrm{C}$ NMR (DMSO- $\left.d_{6}\right): \delta 76.5$, 121.7, 138.6, 149.4, 163.8, 171.8; MS m/z (\%):243 [M $]$ (100), 243 (70), 226 (163), 160 (202), 144 (13), 117 (20.1), 90 (60), 54 (100). Anal. Calcd for $\mathrm{C}_{11} \mathrm{H}_{9} \mathrm{~N}_{5} \mathrm{O}_{2}$ (243.22): C, 54.32; H, 3.73; N, 28.79. Found: C, 54.36; H, 3.70; N, 28.74.

\section{Reaction of 2,6-Bis(3-oxo-3-propanenitrile) pyridine(2) with the hydrazonoyl halide derivatives. General procedure}

3-Oxopropanenitriles 2 ( $0.42 \mathrm{~g}, 2 \mathrm{mmol})$ was added to a stirred ethanolic sodium ethoxide solution [prepared from sodium metal $(0.11 \mathrm{~g})$ and absolute ethanol $(20 \mathrm{~mL})]$. After stirring for $20 \mathrm{~min}$, the appropriate hydrazonoyl halide $9 \mathbf{a}-\mathbf{g}(4 \mathrm{mmol})$ was added and the reaction mixture was stirred at room temperature for $14 \mathrm{~h}$. The formed solid was collected by filtration, washed with water, dried and finally recrystallized from the appropriate solvent, to afford the corresponding pyrazole pyridine derivatives 11a-g. 
2,6-Bis[3-acetyl-4-cyano-1-phenyl-1 $H$-pyazol-5-yl]pyridine (11a). Yield 80\%; yellow micro crystals (from ethanol-dioxan); $\mathrm{mp}>300{ }^{\circ} \mathrm{C}$. IR (KBr): $v 1671(\mathrm{C}=\mathrm{O}), 2210(\mathrm{CN}) \mathrm{cm}^{-1} .{ }^{1} \mathrm{H}$ NMR $\left(\mathrm{DMSO}-d_{6}\right): \delta 2.5\left(\mathrm{~s}, 6 \mathrm{H}, 2 \mathrm{CH}_{3}\right.$-acetyl) $7.34(\mathrm{~m}, 10 \mathrm{H}$, ArH's), 8.03(m, 3H, pyridine). MS $\mathrm{m} / \mathrm{z}$ (\%):497 (M $\mathrm{M}^{+}$(30), 496 (10), 395 (30), 298 (51), 191 (54), 93 (100), 77 (25). Anal. Calcd for $\mathrm{C}_{29} \mathrm{H}_{19} \mathrm{~N}_{7} \mathrm{O}_{2}$ (497.51): C, 70.01; H, 3.85; N, 19.71. Found C, 70.04; H, 3.81; N, 19.74

2,6-Bis[3-acetyl-4-cyano-1-1H-pyazol-p-tolyl 5-yl]pyridine (11b). Yield 83\%; green solid (from ethanol/dioxan); $\mathrm{mp}>300{ }^{\circ} \mathrm{C}$. IR $(\mathrm{KBr}): v 2198(\mathrm{CN}), 1655(\mathrm{C}=\mathrm{O}) \mathrm{cm}^{-1} .{ }^{1} \mathrm{H}$ NMR

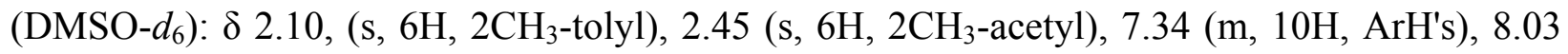
(m, 3H, pyridine). MS m/z (\%): $525\left[\mathrm{M}^{+}\right]$(30), 524 (14), 313 (10), 174 (17), 121 (28), 92 (20), 57 (100). Anal. Calcd for $\mathrm{C}_{31} \mathrm{H}_{23} \mathrm{~N}_{7} \mathrm{O}_{2}$ (525.56): C, 70.84; H, 4.41; N, 18.66. Found: $\mathrm{C}, 70.80 ; \mathrm{H}$, $4.38 ; \mathrm{N}, 18.62$.

2,6-Bis[3-acetyl-1-(4-chloropheny)-4-cyano-1H-pyazol-5-yl]pyridine (11c). Yield 85\%; yellow solid (Ethanol); mp 238-240 ${ }^{\circ} \mathrm{C}$. IR (KBr): v $2237(\mathrm{CN}), 1678(\mathrm{C}=\mathrm{O}) \mathrm{cm}^{-1} .{ }^{1} \mathrm{H}$ NMR

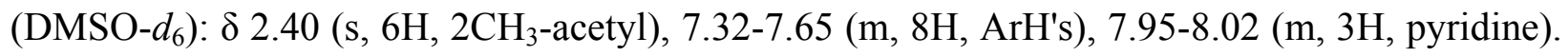
MS m/z (\%): $565\left(\mathrm{M}^{+}\right]$(35), 540 (61), 499 (33), 414 (16), 346 (68), 239 (45), 152 (78), 111 (100). Anal. Calcd for $\mathrm{C}_{29} \mathrm{H}_{17} \mathrm{Cl}_{2} \mathrm{~N}_{7} \mathrm{O}_{2}$ (566.08): C, 61.50; H, 3.03; N, 17.31. Found: C, 61.54; H, 3.06; N, 17.34.

2,6-Bis[4-cyano-3-ethoxycarbonyl-1-phenyl-1H-pyrazol-5-yl]pyridine (11d). Yield 75\%; Yellow needles (from ethanol); mp 234-235 ${ }^{\circ} \mathrm{C}$. IR (KBr): v $2184(\mathrm{CN}), 1725(\mathrm{C}=\mathrm{O}) \mathrm{cm}^{-1} .{ }^{1} \mathrm{H}$ NMR (DMSO- $\left.d_{6}\right): \delta 1.32\left(\mathrm{t}, 6 \mathrm{H}, 2 \mathrm{CH}_{3}\right), 4.39\left(\mathrm{q}, 4 \mathrm{H}, 2 \mathrm{CH}_{2}\right), 7.34-7.56(\mathrm{~m}, 10 \mathrm{H}, \mathrm{ArH}$ 's), 7.908.03 (m, 3H, pyridine). MS m/z (\%):557 ( $\left.\mathrm{M}^{+}\right]$(35), 556 (49), 510 (28), 242 (16), 206 (17), 77 (100). Anal. Calcd for $\mathrm{C}_{31} \mathrm{H}_{23} \mathrm{~N}_{7} \mathrm{O}_{4}$ (557.56): C, 66.78; H, 4.16; N, 17.59. Found: C, 66.74; H, 4.19; N, 17.63 .

2,6-Bis[4-cyano-3-ethoxycarbonyl-1H-pyrazol-1-tolyl-5-yl]pyridine (11e). Yield $78 \%$; Yellow micro crystals (from ethanol/dioxan); mp > $300^{\circ} \mathrm{C}$. IR (KBr): $v 2263(\mathrm{CN}), 1721(\mathrm{C}=\mathrm{O})$

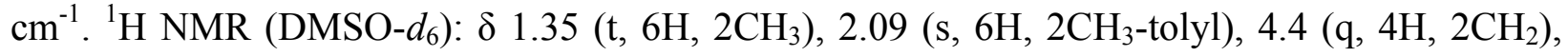
7.25-7.46 (m, 8H, ArH's), 7.69-8.10 (m, 3H, pyridine). MS m/z (\%): $585\left(\mathrm{M}^{+}\right]$(38). Anal. Calcd for $\mathrm{C}_{33} \mathrm{H}_{27} \mathrm{~N}_{7} \mathrm{O}_{4}(585.61)$ : C, 67.68; H, 4.65; N, 16.74. Found: $\mathrm{C}, 67.65 ; \mathrm{H}, 4.61 ; \mathrm{N}, 16.70$.

2,6-Bis[1-(4-chlorophenyl)-4-cyano-3-ethoxycarbonyl-1 $H$-pyrazol-5-yl]pyridine (11f)

Yield 80\%; Yellow solid (from ethanol/dioxan); mp > $300{ }^{\circ} \mathrm{C}$. IR (KBr): $v 2162(\mathrm{CN}), 1742$ $(\mathrm{C}=\mathrm{O}) \mathrm{cm}^{-1} .{ }^{1} \mathrm{H}$ NMR (DMSO-d $): \delta 1.37\left(\mathrm{t}, 6 \mathrm{H}, 2 \mathrm{CH}_{3}\right), 4.12\left(\mathrm{q}, 4 \mathrm{H}, 2 \mathrm{CH}_{2}\right), 7.34-7.41(\mathrm{~m}, 10 \mathrm{H}$, ArH's), 7.65-7.95 (m, 3H, pyridine). ${ }^{13} \mathrm{C}$ NMR (DMSO- $\left.d_{6}\right): \delta 13.7,63.2,117.3,120.0,121.1$, 129.2, 130.8, 140.1, 141.4, 156.5, 157.3, 159.1. MS m/z (\%): $625\left(\mathrm{M}^{+}\right]$(38), 624 (21), 592 (23), 510 (31), 467 (36), 284 (64), 210 (100), 89 (98). Anal. Calcd for $\mathrm{C}_{31} \mathrm{H}_{21} \mathrm{Cl}_{2} \mathrm{~N}_{7} \mathrm{O}_{4}$ (626.45): C, 59.44; H, 3.83; N, 15.65. Found: C, 59.47; H, 3.87; N, 15.61.

2,6-Bis[4-cyano-3-phenylcarbamoyl-1-phenyl-1H-pyrazol-5-yl]pyridine(11g). Yield 89\%; Yellow solid (from ethanol/dioxan); mp 285-286 ${ }^{\circ} \mathrm{C}$. IR (KBr): $v 3390$ (NH), 2238 (CN) $1689(\mathrm{C}=\mathrm{O}) \mathrm{cm}^{-1} .{ }^{1} \mathrm{H}$ NMR (DMSO-d $): \delta$ 7.15-7.36 (m, 20H, ArH's), 7.82-8.15 (m, 3H, pyridine), 10.61 (s,2H, $\mathrm{D}_{2} \mathrm{O}$ exchangeable, 2NH). $\mathrm{MS} m / z(\%): 651\left(\mathrm{M}^{+}\right](17), 559(8), 522$ (15), 
393 (15), 340 (13), 119 (20), 77 (00). Anal. Calcd for $\mathrm{C}_{39} \mathrm{H}_{25} \mathrm{~N}_{9} \mathrm{O}_{2}(651.21)$ : C, 71.88; H, 3.87; N, 19.34. Found: C, 71.85; H, 3.84; N, 19.30.

\section{2,6-Bis[4-amino-7-methyl-2-phenyl-2H-pyrazolo[3,4-d]pyridazin-3-yl]pyridine (13a).}

Hydrazine hydrate $80 \%(2 \mathrm{ml})$ was added to a solution of compound 11a $(0.25 \mathrm{~g}, 5 \mathrm{mmol})$ in ethanol $(10 \mathrm{~mL})$. The reaction mixture was heated under reflux for $3 \mathrm{~h}$, concentrated in vacuum, cooled, and diluted with water; the precipitate obtained was filtered, washed with ice-cold water, dried and crystallized from ethanol to afford compound 13a. Yield 69\%; yellow powder (from ethanol); mp 285-286 ${ }^{\circ} \mathrm{C}$. IR (KBr): $v$ 3390, $3300\left(\mathrm{NH}_{2}\right) \mathrm{cm}^{-1} .{ }^{1} \mathrm{H}$ NMR (DMSO- $\left.d_{6}\right): \delta 2.13(\mathrm{~s}$, $\left.6 \mathrm{H}, 2 \mathrm{CH}_{3}\right), 4.3$ (s, 4H, 2NH, $\mathrm{D}_{2} \mathrm{O}$ exchangeable,), 7.13-7.35 (m, 10H, ArH's), 7.81-8.13 (m, 3H, pyridine), MS m/z (\%): $525\left(\mathrm{M}^{+}\right](61)$ ), 77 (100). Anal. Calcd for $\mathrm{C}_{29} \mathrm{H}_{23} \mathrm{~N}_{11}$ (525.57): C, 66.27; H, 4.41; N, 29.32. Found: C, 66.33; H, 4.44; N, 29.30.

\section{Acknowledgements}

This research was support by Science and Technology Development Fund-Egypt (project No.170)

\section{References}

1. Elnagdi, M. H.; Elmoghayar, M. R. H.; Elgemeie, G. E. H. Synthesis 1984, 1.

2. Abdel-Khalik, M. M.; Agamya, S. M.; Elnagdi, M. H. Z. Naturforsch. 2000, 55B, 1211.

3. Al-Saleh, B.; El-Apasery, M. A.; Elnagdi, M. H. J. Chem. Res. 2004, 578.

4. El-Dusouqui, O. M. E.; Abdelkhalik, M. M.; Al-Awadi, N. A.; Dib, H. H.; George, B. J.; Elnagdi, M. H. J. Chem. Res. 2006, 295.

5. Hammond, R. J.; Poston, B. W.; Ghiviriga, I.; Feske, B. D. Tetrahedron Lett. 2007, 48, 1217.

6. Aurelio, L.; Figler, H.; Flynn, B. L.; Linden, J.; Scammells, P. J. Bioorg. Med. Chem. 2008, $16,1319$.

7. Al-Awadi, N. A.; Abdelkhalik, M. M.; Abdelhamid, I. A. Synlett. 2007, 2979.

8. Fleming, F. F.; Zhang, Z. Tetrahedron 2005, 61, 747.

9. Burgaz, E. V.; Yilmaz, M.; Pekel, A. T.; Oktemer, A. Tetrahedron 2007, 63, 7229.

10. Lahaus, G.; Dittmar, S. W. Africa Patent 6 906036; Chem. Abstr. 1988, 73, 720308.

11. Youngdale, G. A. U.S. Patent: 4288 440; Chem. Abstr. 1982, 96, 65965

12. Dorigo, P.; Gaion R. M.; Belluco, P.; Fraccarollo, D.; Maragano I.; Bombien, G.; Benelollo, F.; Mostil, L.; Orsini, F. J. Med. Chem., 1993, 36, 2475

13. Dolle, V.; Nguyen, E. C.H.; Aubertin, A.M.; Kirm, A. M.; Andreola, L.; Jamieson, G.; Tarrago-Litvak, L.; Bisagni, E. J. Med. Chem., 1995, 38, 4679.

14. Subbanwad, G.R., Vibhute, Y.B. J. Indian Chem. Soc. 1992, 69, 11, 781. 
15. Teruhisa, N., Hideo, O. J. Phar. Soc. Japan 1956, 76, 390.

16. Farag, A. M; Mayhoub, A. S.; Abdalla, T. M.; Amr, A. E.; Ali, K. A.; Abdel-Hafez, N. A.; Abdalla, M. M. Arch. Pharm. Chem. Life SCI. 2010, 343, in press.

17. Amr, A. E.; Ali, K. A.; Abdalla, M. M. Eur. J. Med. Chem. 2008, 44, 2, 901.

18. Ali, K. A.; Elkholy, Y. M.; Farag, A. M.; J. Heterocycl. Chem. 2008, 45, 1, 279.

19. Ali, K. A.; Elkholy, Y. M.; Farag, A. M.; J. Heterocycl. Chem. 2006, 43, 5, 1183.

20. Palecek, J.; Valihrach, J. Collect. Czech. Chem. Commun. 1981, 46, 748.

21. Gao, Y.; Wang, H.; Xu, M.; Lian, H.; Pan, Y.; Shi, Y. Org. Prep. Proceed. Int. 2001, 33, 351.

22. Ji, Y.; Trenkle, W. C.; Vowles, J. V. A. Org. Lett. 2006, 8, 1161.

23. Herschhorn, A.; Lerman, L.; Weitman, M.; Gleenberg, I. O.; Nudelman, A.; Hizi, A. J. Med. Chem. 2007, 50, 2370.

24. Fleming, F. F.; Iyer, P. S. Synthesis 2006, 893.

25. Elnagdi, M. H.; Elmoghayar, M. R. H.; Fleita, F.D. J.Prakt. Chem. 1974, 316, 975.

26. Elnagdi, M. H.; Elmoghayar, M. R. H.; Hafez, E. A.; Alnima, H. H. J. Org. Chem. 1975, 40, 2604.

27. Dieckmann, W.; Platz, O. Chem. Ber., 1906, 38, 2989.

28. Hegarty, A. F.; Cashoman, M. P.; Scott, F. L. Chem. Commun., 1971, 13, 884.

29. Shawali, A. S.; Abdelhamid, A. O. Tetrahydron, 1971, 27, 2517. 